\title{
Gun trauma and ophthalmic outcomes
}

N Chopra',2, KA Gervasio ${ }^{1,2,3}$, B Kalosza ${ }^{4}$ and $\mathrm{AY} \mathrm{Wu}^{1,2,5}$

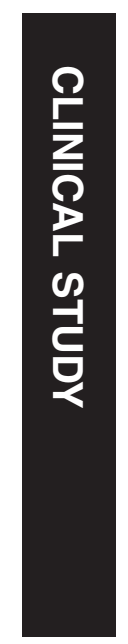

\begin{abstract}
Purpose This retrospective cohort study assesses the visual outcomes of patients who survive gunshot wounds to the head. Methods The Elmhurst City Hospital Trauma Registry and Mount Sinai Data Warehouse were queried for gun trauma resulting in ocular injury over a 16 -year period. Thirty-one patients over 16 years of age were found who suffered a gunshot wound to the head and resultant ocular trauma: orbital fracture, ruptured globe, foreign body, or optic nerve injury. Gun types included all firearms and air guns. Nine patients were excluded due to incorrect coding or unavailable charts. Statistical analysis was performed using a simple bivariate analysis $\left(\chi^{2}\right)$.

Results Of the 915 victims of gun trauma to the head, $27(3.0 \%)$ sustained ocular injuries. Of the 22 patients whose records were accessible, 18 survived. Eight of the 18 surviving patients $(44 \%)$ suffered long-term visual damage, defined as permanent loss of vision in at least one eye to the level of counting fingers or worse. Neither location of injury $(P=0.243)$, nor type of gun used $(P=0.296)$, nor cause of gun trauma $(P=0.348)$ predicted visual loss outcome. The Glasgow Coma Scale eye response score on arrival to the hospital also did not predict visual loss outcome $(P=0.793)$.

Conclusion There has been a dearth of research into gun trauma and even less research on the visual outcomes following gun trauma. Our study finds that survivors of gun trauma to the head suffer long-term visual damage $44 \%$ of the time after injury.
\end{abstract} Eye (2018) 32, 687-692; doi:10.1038/eye.2017.249; published online 22 December 2017

\section{Introduction}

Gun trauma is an important issue that attracts national and international attention. In 2014, 33599 persons died from firearm injuries in the United States, which accounted for $16.8 \%$ of all injury deaths in that year. ${ }^{1}$ Despite the magnitude of this issue, there has been a dearth of research into gun trauma. A 2017 study found that gun trauma kills roughly as many people as sepsis; funding for gun trauma research, however, amounted to $\sim 0.7 \%$ of the funding dedicated to researching sepsis, and the volume of research published on gun trauma was roughly $4 \%$ of the research published on sepsis. ${ }^{2}$

Contributing factors likely include restrictions on collecting and sharing gun data as well as limited funding towards gun research. When performing background checks on gun purchases, for example, any identifying information collected must be destroyed within $24 \mathrm{~h}$ of a decision (28 CFR 25.9-Retention and destruction of records in the system, 1998). Furthermore, legislation specifically prohibits any national registry of firearms, firearms owners, or firearms transactions at the federal level (Firearms Owners' Protection Act, 1986). While there are exceptions-guns reported as stolen, various registries at the state level—there is no national repository for collecting and sharing gun data. At the international level, the United Nations has sought to limit illicit trading of conventional arms, encouraging national recordkeeping requirements and the sharing of such records; however, the treaty specifically reaffirms the right of member states to regulate and control conventional arms exclusively within their own territories, pursuant to their own legal systems (United Nations Arms Trade Treaty, 2013).

In addition to limited information on gun ownership, there is also a lack of federal funding for gun research in the United States as referenced above. The reasons for this are multifaceted but are partly influenced by legislation. For example, in 1996 it was stipulated that 'none of the funds made available for injury prevention and control at the Centers for Disease Control and Prevention may be used to advocate or promote gun control'. ${ }^{3}$

To the extent that there is limited research on gun trauma overall, there is even less research on the ophthalmic outcomes following gun trauma. Furthermore, existing research focuses
${ }^{1}$ Department of Ophthalmology, Icahn School of Medicine at Mount Sinai, New York, NY, USA

${ }^{2}$ Department of Ophthalmology, Elmhurst Hospital Center, Elmhurst, NY, USA

${ }^{3}$ Department of Medicine, Memorial Sloan Kettering Cancer Center, New York, NY, USA

${ }^{4}$ Department of Trauma Research, Elmhurst Hospital Center, Elmhurst, NY, USA

${ }^{5}$ Department of Ophthalmology, Stanford University School of Medicine, Palo Alto, CA, USA

Correspondence: AY Wu, Department of Ophthalmology, Stanford University School of Medicine, 2370 Watson Court, Suite 200, Palo Alto, CA 94303, USA

Tel: +1 6504970758 ;

Fax: +1 6507368232

E-mail: awu1@stanford.edu

Received: 7 March 2017 Accepted in revised form: 4 October 2017

Published online: 22

December 2017 
heavily on deaths from gun trauma; however, gun trauma results in many more nonfatal injuries than deaths, resulting in the potential for years of life lived with disability. ${ }^{4}$ Given the magnitude of this issue, and to increase research in this important area, we explore the effects of gun trauma to assess the visual outcomes of patients who survive gunshot wounds to the head.

\section{Materials and methods}

The Elmhurst City Hospital Trauma Registry and Mount Sinai Data Warehouse were queried for International Classification of Diseases Ninth Revision (ICD-9) and Tenth Revision (ICD-10) codes for gun trauma resulting in ocular injury, and paper charts were obtained to evaluate ophthalmology notes. The study protocol received institutional review board approval from both the Elmhurst Research Committee and Mount Sinai Hospital to conduct a comprehensive paper chart review. The Elmhurst City Hospital is a level 1 trauma center serving a population of approximately one million people throughout the ethnically diverse neighborhoods of northwestern Queens, New York City. Mount Sinai Hospital is a 1183-bed general medical and surgical facility with 102267 emergency department visits in the most recent year reported, serving communities in East Harlem and surrounding Manhattan, New York City. In 2015, there were 50088 violent crimes in New York City. ${ }^{5}$

The Elmhurst City Hospital Trauma Registry and Mount Sinai Data Warehouse were initially queried for all patients presenting with any form of gun trauma between 1 January 2000 and 8 May 2016, using internal record keeping that tracks injuries from guns. Gun types included all firearms and air guns. This data set was further filtered by ICD-specific ocular codes that capture direct and indirect 'injuries to the head' as well as 'diseases of the eye and adnexa' to account for secondary injury such as sequelae from retained foreign bodies (Table 1). All causes of gun trauma were considered, including violence, accidents, and self-harm.

Thirty-one patients who suffered a gunshot wound to the head with resultant ocular trauma were identified having diagnoses including orbital fracture, ruptured globe, foreign body, and optic nerve injury. Of the 31 patients queried, four were excluded due to incorrect coding upon chart review, and five were excluded because their charts were unavailable. Data from the 22 remaining patients were then assessed. We defined longterm visual damage as permanent loss of vision from enucleation/evisceration or traumatic optic neuropathy, resulting in impaired vision to the level of counting fingers or worse. We used a simple bivariate analysis $\left(\chi^{2}\right)$ to evaluate the ability of any given metric to predict longterm visual damage. The study was Health Insurance
Table 1 Ocular trauma codes queried

\begin{tabular}{ll}
\hline ICD-9 code & Description \\
\hline 802 & $\quad$ Closed fracture of nasal bones \\
802.1 & $\quad$ Open fracture of nasal bones \\
802.6 & $\quad$ Closed fracture of orbital floor (blow-out) \\
802.7 & $\quad$ Open fracture of orbital floor (blow-out) \\
802.8 & $\quad$ Closed fracture of other facial bones \\
802.9 & $\quad$ Open fracture of other facial bones \\
$870.0-870.9 \quad$ Open wound of ocular adnexa
\end{tabular}

The Elmhurst City Hospital Trauma Registry and Mount Sinai Data Warehouse were queried for gun trauma cases over a 16-year period; these results were filtered for cases resulting in ocular injury using the ICD codes listed here.

Portability and Accountability Act compliant, with protection of all individually identifiable health information.

\section{Results}

Of 915 patients presenting to the Elmhurst and Mount Sinai Hospital Emergency Departments with injuries due to gun trauma, $27(3 \%)$ sustained ocular injuries. Of the 22 patients whose records were accessible, four were noted to have ocular injury on presentation but then died in the operating room. Blood loss was a major contributing factor in each of these cases, as three of the four patients had been found down after an unknown amount of time 
since their injuries and the fourth patient sustained significant bleeding above the neck. The causes of gun trauma in these four patients were violence (50\%) and self-harm (50\%). Analysis was conducted on the remaining 18 patients.

Of the surviving 18 patients, the median age was 28 years (range, 13-57 years), 15 (83\%) of whom were male. The average Injury Severity Score of patients at presentation was $14.15( \pm 9.69)$. The average Glasgow Coma Scale (GCS) score was $12.85( \pm 4.16)$. On average, the length of stay for each patient was 9.27 days $( \pm 12.31)$. Two patients $(11 \%)$ were shot directly to the globe, 5 (28\%) were shot to the orbit, and $11(61 \%)$ were shot elsewhere to the head. For the cases where data were available, six patients (46\%) were shot with a handgun, four $(31 \%)$ with an air gun, and three $(23 \%)$ with a shotgun. For the cases where data were available, the causes of gun trauma included violence in nine cases $(64 \%)$, accidents in four cases $(29 \%)$, and self-harm in one case $(7 \%)$. Five of the 18 patients $(28 \%)$ sustained bilateral ocular injury, with the remaining 13 (72\%) sustaining injury to just one eye. Ten patients (56\%) suffered wounds beyond their direct ocular injuries, including facial fractures, facial nerve injury, gunshot wounds to the trunk and extremities, and, in the most severe cases, traumatic brain injury. Table 2 summarizes gunshot locations, types of gun involved, injuries, and causes of injury for all 18 patients.

In total, 8 of the 18 surviving patients (44\%) suffered long-term visual damage, defined as permanent loss of vision in at least one eye to the level of counting fingers or worse. Two of these eight patients underwent enucleation in both eyes because of their injuries, five underwent enucleation in one eye, and the eighth patient suffered permanent loss of vision to the level of hand motion in one eye due to traumatic optic neuropathy. The two patients needing bilateral enucleations were the two patients in our study with associated traumatic brain injury from their gunshot wounds. Neither location of injury ( $p=0.243)$, nor type of gun used $(p=0.296)$, nor cause of gun trauma $(p=0.348)$ predicted visual loss outcome. The GCS eye response score on arrival to the hospital also did not predict visual loss outcome $(p=0.793)$. Table 3 summarizes the predictive value of injury location, type of gun used, cause of gun trauma, and GCS eye response score on arrival on long-term vision loss.

\section{Discussion}

There has been a dearth of research into gun trauma and even less research on the visual outcomes following gun trauma. A subset of the research on visual outcomes has focused on accidental gun trauma, particularly in pediatric populations from air and pellet guns. In a Finnish study of 202 children's eye injuries, $9 \%$ of patients were left with a permanent disability; guns, fireworks, tools, and pellet guns were found to be the most dangerous objects. ${ }^{6}$ Haring et $a l^{7}$ found that among sports-related eye injuries in the United States, activities involving projectiles pose the greatest risk for visual impairment in the short term, although in their study long-term outcomes were unavailable.

While these studies are important, they do not address gun trauma due to violence or self-harm in adults. Studies exploring visual outcomes following suicide attempts with guns include Kucuker et $a l^{8}$ who offer a treatment algorithm to restoring visual functionality and appearance following severe maxillofacial and ocular injuries in failed suicide attempts using long-barreled guns. In a case study of two suicide attempts in Germany, via gunshot wounds to the lateral orbit in attempted suicides, one patient presented with a double penetration of both orbits and destruction of both globes, making reconstruction not possible. The other patient presented with multiple shots to the head from a small caliber gun (5.6 $\mathrm{mm})$, where one bullet entered the right orbit behind the globe; the bullet was localized using computed tomography and surgically removed with preservation of the globe and with a postoperative visual acuity of $20 / 60 .^{9}$

Recent research has looked at military injury due to gunshot wounds, comparing outcomes between military and civilian patients. A 2016 study saw important differences regarding the anatomic location, gender, and race distribution of maxillofacial GSWs between the military and civilian populations. For example, the mandible is the most commonly affected region of the maxillofacial skeleton in civilians, while the maxilla is more commonly affected in military gunshot wounds. Military victims are more likely to be white (34\%) and male $(99 \%)$ when compared with their civilian counterparts ( $27 \%$ and $87 \%$, respectively). ${ }^{10}$

In addition to injury from bullets and explosive fragments themselves, injury from blast effects or highenergy transfer in the closed space of the head are important causes of adverse visual outcomes. The effects of indirect injury from blast effects have been noted on several occasions, including blast lung injury, tympanic membrane damage, and even colonic injury, where one study found indirect damage to the colon by propagation of energy by the missile, remote from the track of the projectile. ${ }^{11-13}$

With regards to blast effects in the head specifically, an 11-year, multi-institutional study of 720 patients with gunshot wounds and blast injuries to the face found high mortality and associated morbidity in these cases, with $26 \%$ of patients dying and $67 \%$ of patients hospitalized 


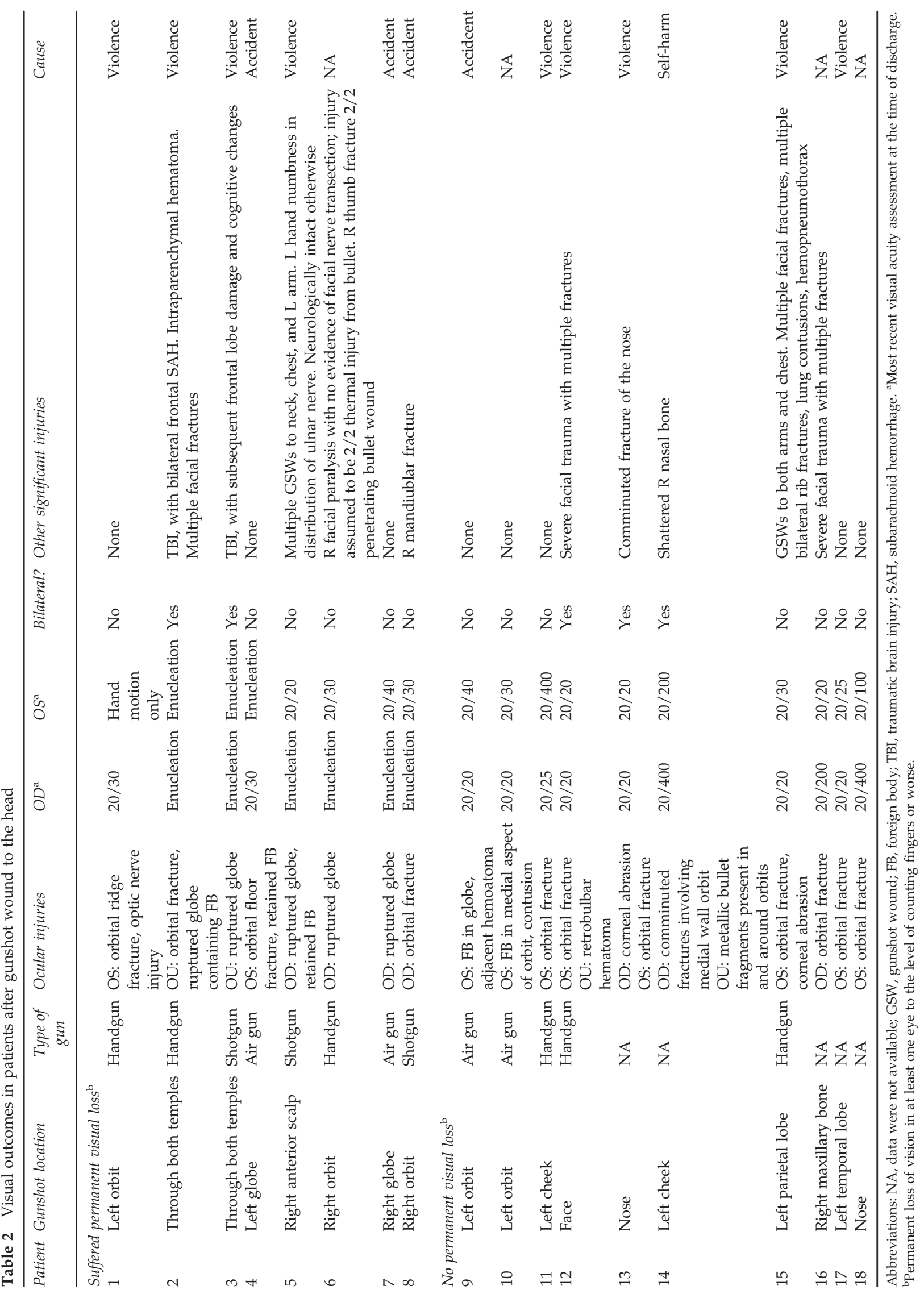


Table 3 Bivariate analysis- $\chi^{2}$

\begin{tabular}{|c|c|c|c|c|}
\hline & Total population $\mathrm{N}=18$ & Long-term vision loss $\mathrm{N}=8$ & No long-term vision loss $\mathrm{N}=10$ & P-value \\
\hline Location of injury, \% (n) & & & & 0.243 \\
\hline Globe & $11.11(2)$ & $25.00(2)$ & $0.00(0)$ & \\
\hline Orbit & $27.28(5)$ & $25.00(2)$ & $30.00(3)$ & \\
\hline Elsewhere & $61.11(11)$ & $50.00(4)$ & $70.00(7)$ & \\
\hline Type of gun, \% (n) & & & & 0.296 \\
\hline Air gun & $30.77(4)$ & $25.00(2)$ & $40.00(2)$ & \\
\hline Handgun & $46.15(6)$ & $37.50(3)$ & $60.00(3)$ & \\
\hline Shotgun & $23.08(3)$ & $37.50(3)$ & $0(0)$ & \\
\hline Cause of gun trauma & & & & 0.348 \\
\hline Violence & $64.29(9)$ & $57.14(4)$ & $71.43(5)$ & \\
\hline Accidents & $28.57(4)$ & $42.86(3)$ & $14.29(1)$ & \\
\hline Self-harm & $7.14(1)$ & $0.00(0)$ & $14.29(1)$ & \\
\hline Arrival GCS, eye, (\%) (n) & & & & 0.793 \\
\hline Open spontaneously & $83.33(10)$ & $80.00(4)$ & $85.71(6)$ & \\
\hline No eye opening & $16.67(2)$ & $20.00(1)$ & $14.29(1)$ & \\
\hline
\end{tabular}

Abbreviation: GCS, Glasgow Coma Scale.

Population statistics. Predictive value of location of injury, type of gun, cause of gun trauma, and Glasgow Coma Scale eye response on arrival on longterm vision loss.

Note: Where totals do not add to 18 , the relevant data were not available.

$>48 \mathrm{~h}^{14}$ In 2015 Rex et al ${ }^{15}$ noted that ocular injury is among the most common terrorist blast morbidities, arising in up to $28 \%$ of survivors, and yet the nature and extent of ocular injury from the primary blast itself, independent of flying debris, has been poorly explored. The study found that over the course of 1 month from exposure to blast injury, various forms of ocular damage arose from corneal edema to retinal detachment with associated vision loss. Importantly, this study highlights the need for close follow-up with patients exposed to blast injuries, as their prognosis may change over time.

One of the more serious complications from blast injury to the head is traumatic brain injury. A 2013 study of visual quality of life in 60 veterans found that individuals with blast-induced traumatic brain injury reported significant decrements in their subjective visual experiences. ${ }^{16}$ An earlier study that explored an individual's useful field of view after traumatic brain injury found greater functional loss of peripheral vision when compared to controls. ${ }^{17}$ As mentioned earlier, two of our patients suffered traumatic brain injury secondary to their gunshot wounds to the head; in one of these patients, this resulted in cognitive decline necessitating specialized care. In terms of visual outcomes, both patients underwent bilateral enucleation due to their injuries.

Through a variety of mechanisms, gun trauma results in significant morbidity and mortality for victims.

Previous studies have explored many different facets of gun trauma, including the effects of direct damage, indirect damage from blast effects, traumatic brain injury, outcomes in military vs civilian populations, causes of gun trauma, and others. However, the magnitude of this issue warrants increased focus on these and other aspects of gun trauma. Investigation into long-term visual outcomes in the setting of gun trauma to the head has been limited; our study seeks to add to this area of research.

Limitations of this study predominantly include small sample size, lack of available data, and skewed patient outcomes. The limitation of small sample size was further compounded when paper charts for 5 of 27 patients were unavailable and therefore unable to be analyzed.

Furthermore, the remaining charts, having been collected over 16 years in paper form, were not consistent in their recording of data; potentially important information such as whether a patient lost consciousness was therefore not able to be considered. Finally, of the eight patients with long-term vision loss, seven underwent an evisceration or enucleation. The findings of our study may be less relevant for victims of gun trauma with ocular involvement who retain their globe. Future studies should include a larger sample size, a greater number of metrics to consider, more patients with indirect injury to the visual system from gun trauma (eg, traumatic brain injury), and more patients without evisceration/ enucleation whose outcomes can be evaluated at various points in time.

As caretakers of patients with gunshot wounds to the head, ophthalmologists should be able to manage these 
difficult cases and council patients with regard to their visual prognosis. Ocular damage from gunshot wounds to the head can arise in many ways, including direct injury to the visual system by projectiles or blast effects, or indirect damage via traumatic brain injury. More research is needed to tease out the most important factors predicting long-term visual damage. Gunshot wounds to the head are deadly, with $18 \%$ of the study patients not surviving their wounds. Our data show that survivors of gun trauma to the head suffer long-term visual damage $44 \%$ of the time after injury. This research helps to quantify the harm from gun trauma as well as encourages increased focus on this important topic.

\section{Summary}

What was known before

- Gun trauma is an important issue that attracts national and international attention and results in significant morbidity and mortality for victims.

- Previous studies have explored many different facets of gun trauma, including the effects of direct damage, indirect damage from blast effects, traumatic brain injury, outcomes in military vs civilian populations, causes of gun trauma, and others.

- However, investigation into long-term visual outcomes in the setting of gun trauma to the head has been limited.

What this study adds

- We find that survivors of gun trauma to the head suffer permanent visual loss $44 \%$ of the time after injury.

\section{Conflict of interest}

The authors declare no conflict of interest.

\section{Acknowledgements}

We would like to acknowledge our colleagues at Elmhurst Hospital Center, Elmhurst NY, for access to their trauma registry database and statistical support. Specifically, we thank George Agriantonis, MD, Anju Galer, MSN, APRN, ANP-BC, and Praise Nesamony. We would also like to acknowledge support from Research to Prevent Blindness, Inc. and the National Eye Institute (P30-EY026877).

\section{References}

1 Xu J, Murphy SL, Kochanek KD, Bastian BA. Deaths. Final data for 2013. Natl Vital Stat Rep 2016; 64(2): 1-119.
2 Stark DE, Shah NH. Funding and publication of research on gun violence and other leading causes of death. JAMA 2017; 317(1): 84-85.

3 House US. Omnibus Consolidated Appropriations Act, 1997. Departments of Commerce J, and State, the Judiciary, and Related Agencies. Government Publishing Office: Washington, DC, 1997.

4 Fahimi J, Larimer E, Hamud-Ahmed W, Anderson E, Schnorr CD, Yen I et al. Long-term mortality of patients surviving firearm violence. Inj Prev 2016; 22(2): 129-134.

5 Newsday. Major crime in New York City, 2009-2015 2016 (updated 31 August 2016). Available at: http://data. newsday.com/long-island/data/crime/new-york-citycrime-rate/.

6 Haavisto AK, Sahraravand A, Holopainen JM, Leivo T. Paediatric eye injuries in Finland-Helsinki eye trauma study. Acta Ophthalmol 2016; 95(4): 392-399.

7 Haring RS, Sheffield ID, Canner JK, Schneider EB. Epidemiology of sports-related eye injuries in the United States. JAMA Ophthalmol 2016; 134(12): 1382-1390.

8 Kucuker I, Simsek T, Keles MK, Yosma E, Aksakal IA, Demir A. Our treatment approaches in severe maxillofacial injuries occurring after failed suicide attempts using longbarreled guns. J Craniofac Surg 2016; 27(2): e133-e138.

9 Schargus M, Kawa D, Elling M, Kunkel M. Management of suicidal orbital gunshot wounds to the temple. Ophthalmologe 2014; 111(10): 965-969.

10 Guevara C, Pirgousis P, Steinberg B. Maxillofacial gunshot injuries: a comparison of civilian and military data. J Oral Maxillofac Surg 2016; 74(4): 795 e1-795 e7.

11 MacFadden LN, Chan PC, Ho KH, Stuhmiller JH. A model for predicting primary blast lung injury. J Trauma Acute Care Surg 2012; 73(5): 1121-1129.

12 Waterworth TA, Carr MJ. Report on injuries sustained by patients treated at the Birmingham General Hospital following the recent bomb explosions. BMJ 1975; 2(5961): 25-27.

13 Webster C, Mercer S, Schrager J, Carrell TW, Bowley D. Indirect colonic injury after military wounding: a case series. J Trauma 2011; 71(5): 1475-1477.

14 Shackford SR, Kahl JE, Calvo RY, Kozar RA, Haugen CE, Kaups KL et al. Gunshot wounds and blast injuries to the face are associated with significant morbidity and mortality: results of an 11-year multi-institutional study of 720 patients. J Trauma Acute Care Surg 2014; 76(2): 347-352.

15 Rex TS, Reilly MA, Sponsel WE. Elucidating the effects of primary blast on the eye. Clin Exp Ophthalmol 2015; 43(3): 197-199.

16 Lemke S, Cockerham GC, Glynn-Milley C, Cockerham KP. Visual quality of life in veterans with blast-induced traumatic brain injury. JAMA Ophthalmol 2013; 131(12): 1602-1609.

17 Fisk GD, Novack T, Mennemeier M, Roenker D. Useful field of view after traumatic brain injury. J Head Trauma Rehabil 2002; 17(1): 16-25. 